\title{
Remplissages quaternaires et paléohydrologie des vallées sèches d'Ajoie (Jura tabulaire, Suisse)
}

\author{
Luc Braillard, Fribourg
}

\section{Introduction}

Située dans le nord-ouest de la Suisse, l'Ajoie est une région largement ouverte sur la France, qui appartient au district de Porrentruy, dans le canton du Jura. Les travaux de construction de l'autoroute A16 ont permis d'y relancer la recherche dans le domaine de la géologie du Quaternaire et de la géomorphologie. Ces disciplines avaient en effet été délaissées depuis les travaux de LiÈvre (1955), ERZINGER (1943) ou encore Perronne (1955). Durant les deux dernières décennies, une grande quantité d'informations a surgi des études géotechniques, géologiques, hydrogéologiques et pédologiques liées à l'A16. Les campagnes de sondages archéologiques menées par la Section d'archéologie et paléontologie de l'Office de la culture du Canton du Jura et la fouille des sites archéologiques qui en a résulté ont également apporté des données stratigraphiques de grande qualité. C'est dans ce contexte qu'a germé le projet d'une étude multidisciplinaire axée sur la morphogenèse des vallées sèches qui caractérisent le paysage ajoulot (BRAILLARD 2006). Le présent article se concentre sur l'étude des remplissages quaternaires qui colmatent les vallées sèches situées à l'ouest de Porrentruy, dans la région de Chevenez.

D'un point de vue structural, l'Ajoie est rattachée au Jura tabulaire (Fig. 1). La région marque la transition entre les contreforts du Jura plissé au sud et les plaines du Sundgau au nord, prolongation méridionale du Fossé rhénan. Le substrat y est constitué de bancs calcaires d'âge jurassique disposés plus ou moins horizontalement. Du point de vue hydrologique, l'Ajoie présente la particularité d'appartenir au bassin versant du Rhône, alors que c'est la contrée de Suisse la plus proche du Fossé rhénan (région bâloise exceptée). Une grande partie de l'écoulement des eaux est toutefois réalisée en milieu souterrain karstique. Seuls quatre cours d'eau superficiels parcourent la région: le Gland (sur territoire français), l'Allaine, la Coeuvatte et la Vendline. Tous les autres sillons qui apparaissent sur la carte en relief de l'Ajoie (Fig. 2) sont des vallées sèches, c'est-à-dire des vallées au fond desquelles on ne rencontre aucun écoulement superficiel, ou alors un écoulement temporaire seulement. Ces vallées sont particulièrement bien visibles sur le bord du plateau de Bure en rive gauche de l'Allaine, ainsi qu'au sud de Porrentruy où elles entaillent les contreforts du Jura plissé selon une direction subméridienne. La vallée sèche principale de la Haute-Ajoie, qui court de Grand-fontaine à Porrentruy selon une direction ouest-est, est quant à elle plus évasée et à fond plat. Elle est parfois envahie par le Creugenat, cours d'eau temporaire qui surgit occasionnellement d'un gouffre karstique situé à l'est de Chevenez (Fig. 3).

Les vallées sèches d'Ajoie situées en position géomorphologique basse présentent un fond plat qui trahit la présence d'un remplissage quaternaire. C'est le cas notamment de toute la vallée sèche de la Haute-Ajoie, entre Rocourt et Porrentruy, ainsi que des parties aval de ses nombreuses petites vallées sèches affluentes. Actuellement, aucun processus sédimentaire n'est à même d'expliquer ni la présence de ce remplissage ni le type d'érosion qui a conduit au façonnement des vallées sèches qui le contiennent. D'où l'intérêt de reconstituer les conditions de dépôt qui prévalaient lors du colmatage de ces vallées aujourd'hui sèches.

\section{Méthodes}

Les données de terrain, acquises entre 1998 et 2003, proviennent de près de 500 sondages (longueur: $6 \mathrm{~m}$, largeur: $1,6 \mathrm{~m}$, profondeur maximale: $5 \mathrm{~m}$ ) creusés à l'aide d'une pelle mécanique entre Chevenez et Courtedoux dans le cadre de campagnes de prospection archéologique sur le tracé de l'autoroute A16. Ces sondages ont ensuite été complétés par de grandes coupes stratigraphiques (Fig. 4) relevées sur cinq sites archéologiques localisés au fond de la vallée sèche principale de la Haute-Ajoie ou au débouché de petites vallées sèches latérales (longueur totale cumulée des coupes: $1005 \mathrm{~m}$ ). Afin de préciser le diagnostic de terrain relatif à la genèse des couches, des analyses sédimentologiques (granulométrie, étude des résidus de tamisage, morphoscopie) et géochimiques (taux de carbonates, de matière organique, d'humus, de phosphates et $\mathrm{pH}$ ) ont été effectuées au Laboratoire de Préhistoire de l'Université de Bâle (IPNA) sur une centaine d'échantillons prélevés en vrac. Une étude micromorphologique (étude du sédiment meuble au microscope optique polarisant) a également été effectuée sur une cinquantaine de lames minces fabriquées par THOMAs BECKMANN à Braunschweig (Allemagne), à partir de blocs de sédiments non perturbés prélevés dans les coupes stratigraphiques puis indurés par une résine synthétique. On trouvera la localisation précise des sondages et sites archéologiques ainsi que des détails sur les levés 


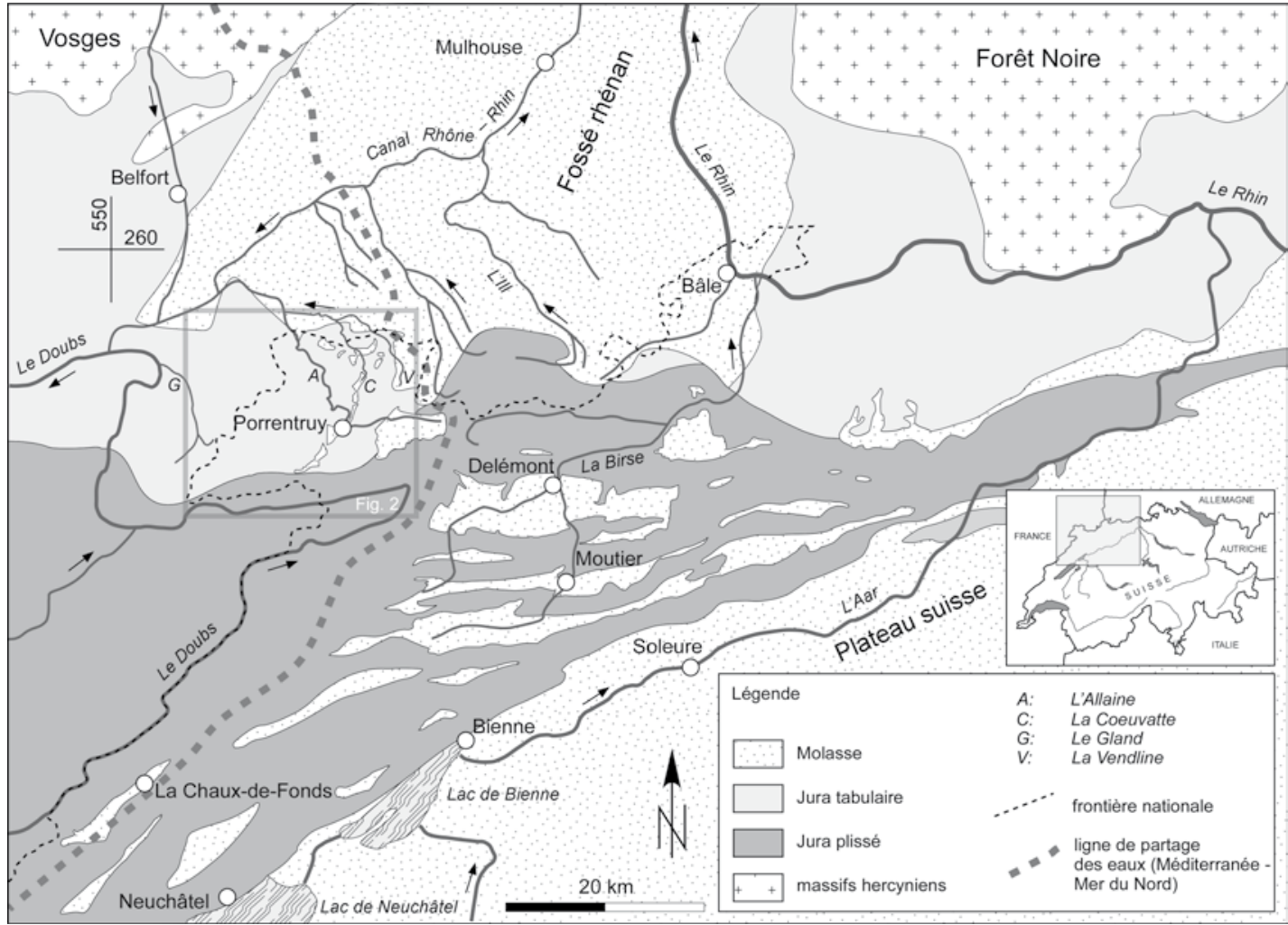

Fig. 1: Cadre géographique et structural du Jura septentrional, situant le Jura tabulaire d'Ajoie (région de Porrentruy, voir Fig. 2) délimité par le Jura plissé au sud et à l'est et par le fossé rhénan au nord Geographical and structural context of the northern Jura chain, locating the tabular Jura of Ajoie (Porrentruy region, see Fig. 2) Geologischer und geographischer Kontext der nördlichen Jurakette mit der Lokalisation des Tafeljuras der Ajoie (Gebiet von Porrentruy, siehe Fig. 2)

Concept et cartographie par L. Braillard, sur la base de la carte tectonique de la Suisse au 1:500'000 (2ème édition, 1980; publiée par la Commission géologique suisse)

stratigraphiques et les méthodes d'analyse dans l'étude de BRAILlARD (2006). Les datations radiocarbone proviennent de mesures AMS réalisées au Laboratoire de l'Université d'Uppsala (Suède), calibrées à partir de la courbe IntCal98 (STUIVER et al. 1998). Des datations OSL ont également été réalisées au Département de Géographie de l'Université de Cologne (Allemagne), sur la fraction 4-11 microns de sédiments lœssiques.

\section{Résultats et discussion}

\subsection{Lithostratigraphie et chronostratigraphie}

Les remplissages quaternaires des vallées sèches de l'Ajoie sont épais de 2 à $10 \mathrm{~m}$. Ils peuvent être subdivisés en dix ensembles chronostratigraphiques (E1-E10, Fig. 5).
L'ensemble 1 correspond à l'horizon agricole actuel. Il est épais de $25 \mathrm{~cm}$ et constitué d'un silt faiblement argileux de teinte brun foncé, humifère et décarbonaté.

L'ensemble 2 regroupe plusieurs couches humifères de teinte brun-jaune, réparties selon trois faciès principaux: silteux, silto-sableux et graveleux. Les faciès fins correspondent à des alluvions déposées au Moyen Age, principalement durant sa phase précoce (Haut Moyen Age: environ 500-1'000 AD). Les faciès graveleux correspondent soit à des colluvions grossières, que l'on retrouve alors en pied de pente, soit à des alluvions, comme dans la plaine du Creugenat. L'épaisseur de E2 est très variable: de 10 à $160 \mathrm{~cm}$.

Composées de silts humifères légèrement argileux 


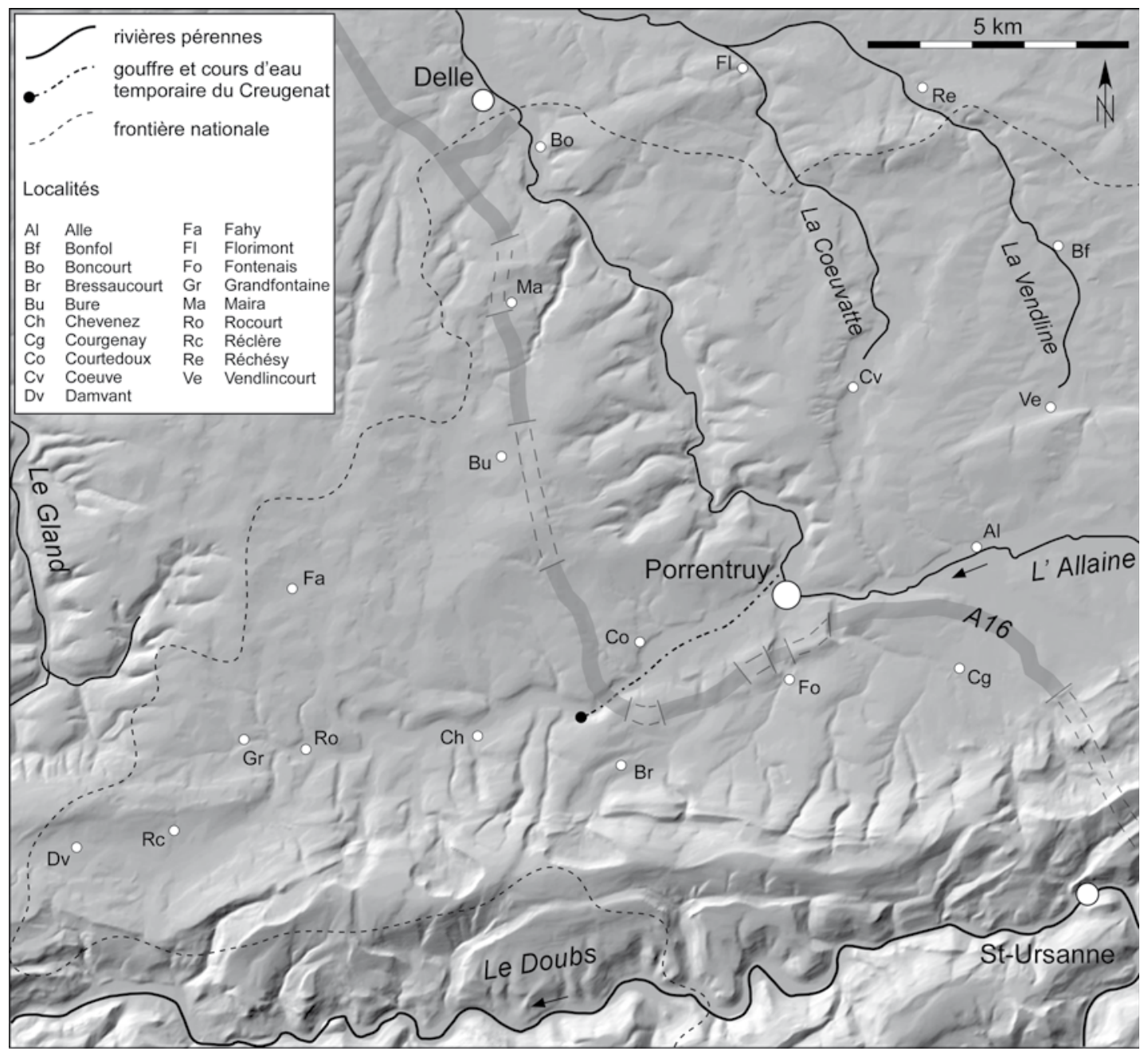

Fig. 2: Carte en relief de l'Ajoie

$3 D$ map of Ajoie

$3 D$-Karte der Ajoie

Reproduit avec l'autorisation de swisstopo (BA081240)

brun-gris à la base desquels (ou dans lesquels) s'intercalent des faciès graveleux à matrice également humifère, les couches de l'ensemble 3 témoignent d'un important impact anthropique sur la dynamique sédimentaire. Riches en paillettes et fragments de charbons de bois issus de défrichements, elles correspondent selon toute vraisemblance aux premières pratiques agricoles dans la région. Mises en place par colluvionnement ou alluvionnement, elles contiennent la plupart des vestiges archéologiques découverts qui s'échelonnent de la fin du Néolithique (Campaniforme) jusqu'au Second Age du Fer (La Tène). Les datations ${ }^{14} \mathrm{C}$ réalisées sur les charbons de bois inclus dans les couches sont centrées sur la Protohistoire, deux résultats remontant au Néolithique final et un au Néolithique moyen. Les couches de E3 couvrent donc chronologiquement la période qui va de la fin du Néolithique à la période gallo-romaine, cette dernière étant toutefois marquée par une sédimentation réduite. Son épaisseur varie généralement entre 20 et $50 \mathrm{~cm}$.

L'ensemble 4 correspond à des limons lœssiques rema- 


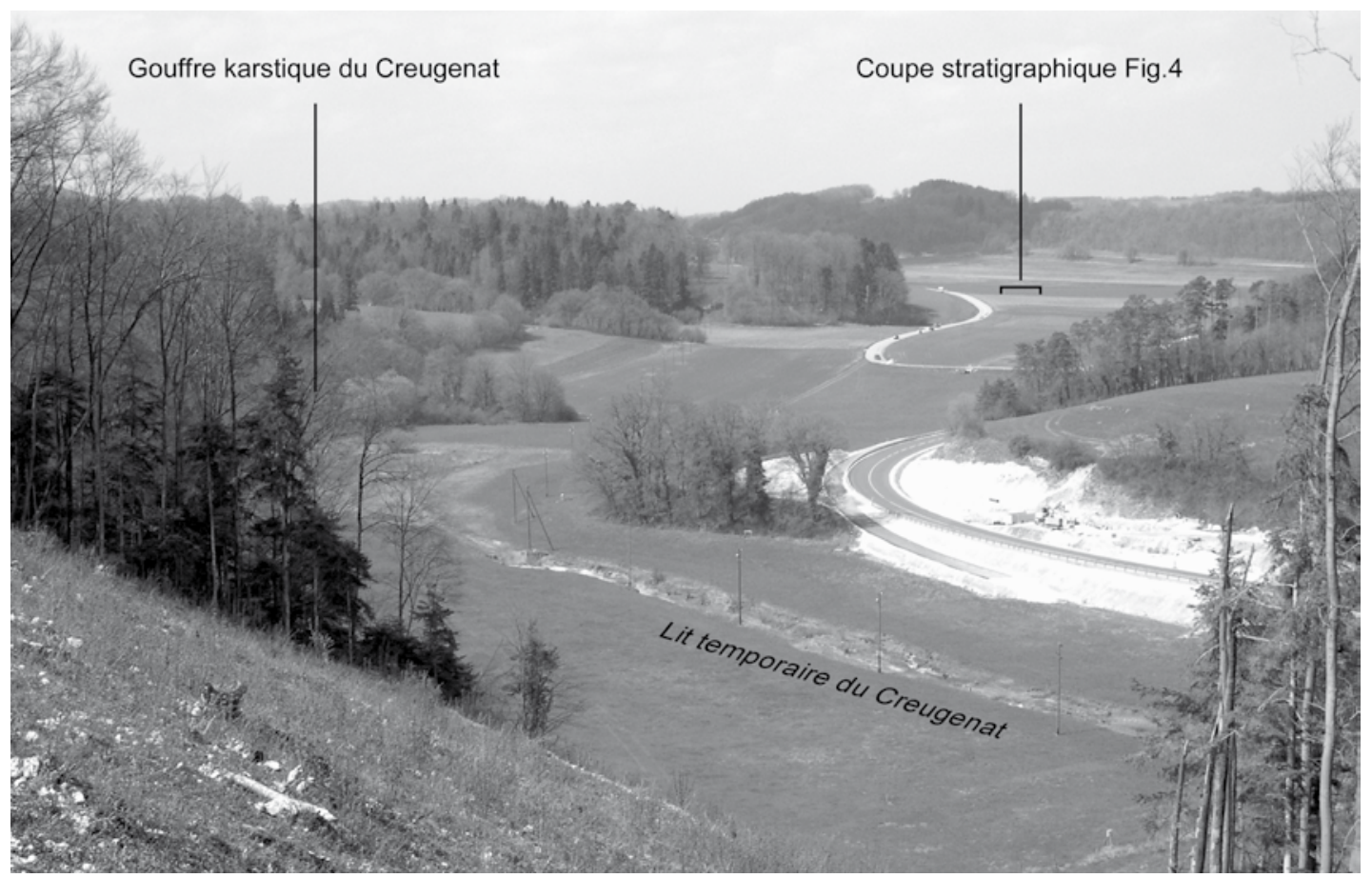

Fig. 3: Vue de la vallée sèche principale de la Haute-Ajoie entre Courtedoux et Chevenez View of the main dry valley of Haute-Ajoie between Courtedoux and Chevenez Ansicht des Haupttrockentals der Haute-Ajoie zwischen Courtedoux und Chevenez Photo: L. BRAILLARD

niés à partir de dépôts éoliens plus anciens. Certaines couches, silteuses et de texture massive, sont encore très proches du faciès lœssique d'origine, alors que d'autres ont été fortement affectées par la pédogenèse. Très argileuses, de teinte brun-ocre et de structure polyédrique, ces dernières correspondent alors à l'horizon argilique (Bt) d'un sol brun lessivé (luvisol). Tant le faciès sédimentaire (lœss remaniés), la pédogenèse (horizon argilique) que la géométrie des couches (solifluxion, ruissellement) s'accordent avec le modèle de pédogenèse proposé par VAN VLIET-LANÖ̈ et al. (1992), qui situe la phase de décarbonatation et de pédogenèse principale du pédocomplexe récent au Tardiglaciaire (Bølling) plutôt qu'à l'Holocène (Atlantique). E4 couvre principalement le Tardiglaciaire ainsi que le tout début de l'Holocène. Sa puissance peut atteindre plus de deux mètres.

Deux faciès graveleux constituent l'ensemble 5: des graviers arrondis hétérométriques en fond de vallée et des graviers anguleux bien calibrés en pied de pente. Les premiers sont des alluvions qui sont lithostratigra- phiquement similaires aux graviers E9. La présence de blocs plus nombreux et plus gros (diamètre maximal: $60 \mathrm{~cm}$ ) indique toutefois un caractère torrentiel plus marqué pour E5 que pour E9. Les seconds s'apparentent à des grèzes litées, constituées pour moitié de petits gélifracts anguleux et aplatis (1-4 cm de diamètre) stratifiés dans le sens de la pente, emballés dans une matrice lœssique carbonatée de type E6. Sur la base de datations OSL et ${ }^{14} \mathrm{C}$, on peut attribuer ces deux faciès au Pléniglaciaire supérieur weichsélien (stade isotopique (SI) 2). L'épaisseur maximale observée de $\mathrm{E} 5$ est de deux mètres.

L'ensemble 6/7 regroupe deux faciès lœssiques: au sommet (E6), des silts jaune-brun clair à verdâtres, presque toujours décarbonatés, qui présentent souvent une structure lamellaire de gel; à la base (E7), des silts argileux brun-rouge toujours décarbonatés, dont la structure a une tendance polyédrique. Du point de vue minéralogique, tant E6 que E7 sont composés essentiellement de minéraux allochtones par rapport au substrat calcaire (quartz dominant, micas clairs, 


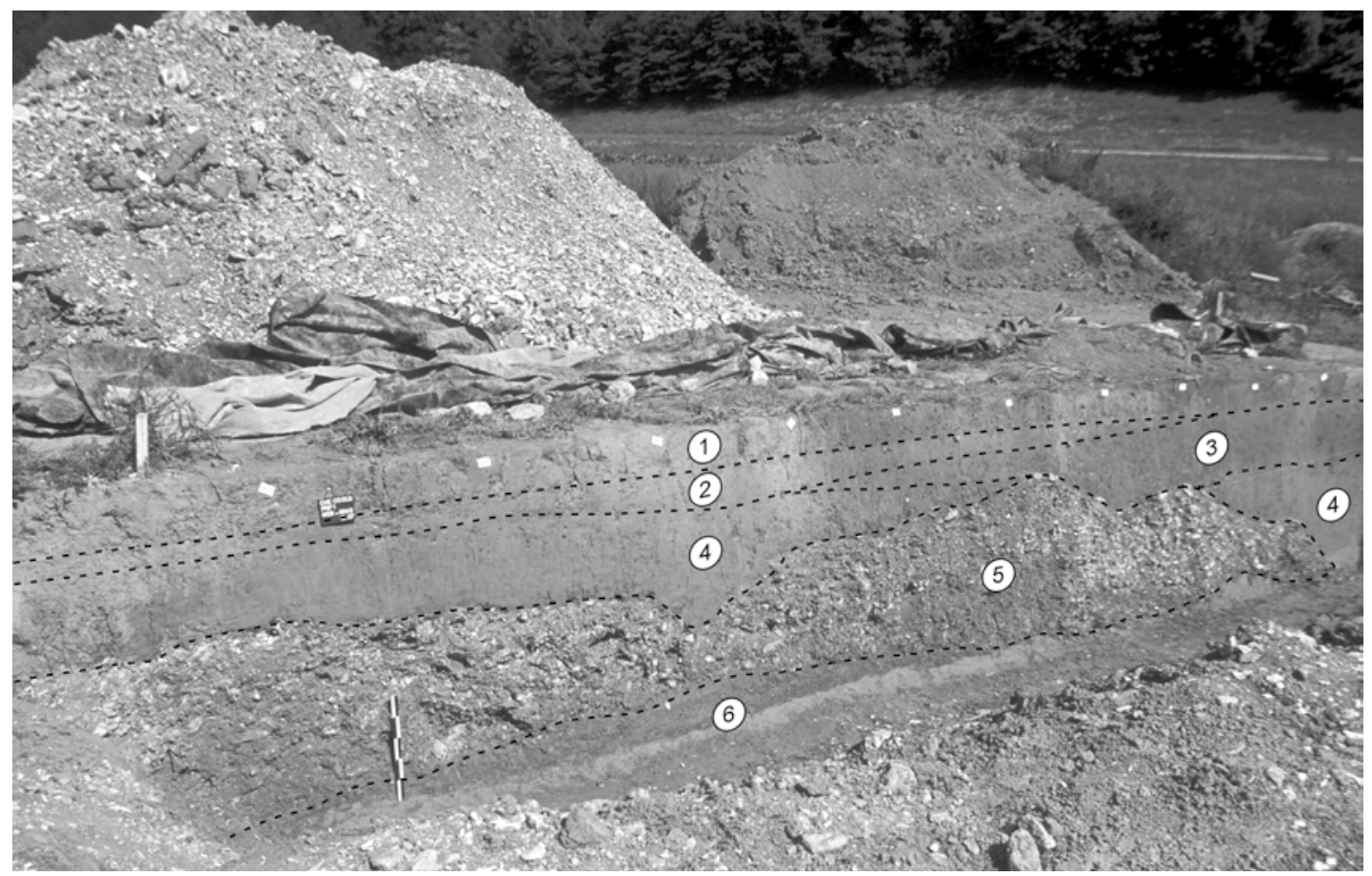

Fig. 4: Exemple d'une partie de coupe stratigraphique (longueur: $11 \mathrm{~m}$ ) réalisée en amont du Creugenat sur le site archéologique de Chevenez, Lai Coiratte (les numéros correspondent aux ensembles stratigraphiques) Example of part of a stratigraphic section (11 $\mathrm{m}$ in length), located upstream from the Creugenat at the archaeological site of Chevenez, Lai Coiratte (numbers refer to stratigraphic units)

Beispiel eines Profilabschnitts (Länge: $11 \mathrm{~m}$ ), aufgenommen flussaufwärts von Creugenat an der archäologischen Grabungsstelle Chevenez, Lai Coiratte (die Nummern bezeichnen stratigraphische Einheiten)

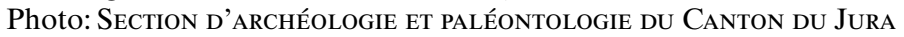

feldspaths). Les datations OSL réalisées dans ces niveaux ont livré des âges qui s'insèrent dans le Pléniglaciaire moyen (SI 3). Exceptionnellement développé en un endroit, à l'amont du gouffre du Creugenat où il atteint deux mètres de puissance, l'ensemble 6/7 se réduit ailleurs à des niveaux discontinus et déformés par solifluxion qui ne dépassent pas $50 \mathrm{~cm}$ d'épaisseur.

L'ensemble 8 n'a été rencontré que dans une seule vallée sèche. Il présente des caractéristiques intermédiaires entre une grèze litée (à la base) et une nappe de solifluxion (au sommet). En raison de sa position stratigraphique sous les loess ruisselés du Pléniglaciaire moyen (E6) et sur les graviers supposés Glaciaire ancien (E9), un âge Pléniglaciaire inférieur (SI 4) peut être proposé pour la mise en place de cette nappe. Au maximum de son développement, E8 atteint $120 \mathrm{~cm}$ d'épaisseur.
Les graviers rattachés à l'ensemble 9 constituent systématiquement le comblement basal des vallées sèches étudiées. Ils sont constitués pour 80 à $90 \%$ d'éléments calcaires relativement bien triés (diamètre maximal: $25 \mathrm{~cm}$ ) et pour 10 à $20 \%$ de matrice silto-argileuse jaune-brun. Les éléments sont jointifs, subanguleux à subarrondis, le plus souvent aplatis. La stratification, peu marquée, est subhorizontale. L'imbrication des éléments aplatis indique une direction du courant vers l'aval. Du point de vue de leur mise en place, ces graviers sont intermédiaires entre des dépôts torrentiels et des dépôts fluviatiles, faciès dont ils mélangent les caractéristiques. Du point de vue chronostratigraphique, ils sont assurément plus anciens que le Pléniglaciaire moyen, puisqu'ils sont coiffés par des loess datés par OSL de cette période. Cependant, aucune datation absolue n'étant disponible pour l'instant, leur âge reste encore sujet à discussion: si l'on considère le niveau d'argiles décarbonatées rencontré localement à leur 


\begin{tabular}{|c|c|c|c|c|}
\hline \multicolumn{3}{|c|}{ CHRONOSTRATIGRAPHIE } & \multicolumn{2}{|c|}{ LITHOSTRATIGRAPHIE } \\
\hline $\begin{array}{l}\mathrm{SI} \\
\text { (Ka) }\end{array}$ & Moderne & E1 & & $\begin{array}{l}\text { Horizon } \\
\text { agricole }\end{array}$ \\
\hline \multirow[t]{2}{*}{1} & Moyen Age & E2 & & \multirow{2}{*}{$\begin{array}{l}\text { Colluvions et } \\
\text { alluvions } \\
\text { humifères }\end{array}$} \\
\hline & $\begin{array}{c}\text { Protohistoire } \\
\text { (Néol. final à Gallo-romain) }\end{array}$ & E3 & & \\
\hline \multirow{2}{*}{$\begin{array}{c}10 \\
2\end{array}$} & $\begin{array}{l}\text { Tardiglaciaire à } \\
\text { Holocène ancien }\end{array}$ & E4 & & $\begin{array}{l}\text { Loess } \\
\text { remaniés } \\
\text { et altérés }\end{array}$ \\
\hline & $\begin{array}{l}\text { Pléniglaciaire } \\
\text { supérieur }\end{array}$ & E5 & & $\begin{array}{l}\text { Alluvions et } \\
\text { grèzes litées }\end{array}$ \\
\hline 3 & $\begin{array}{l}\text { Pléniglaciaire } \\
\text { moyen }\end{array}$ & $\mathrm{E} 6 / 7$ & & $\begin{array}{c}\text { Loess } \\
\text { ruisselés } \\
+/ \text { - altérés }\end{array}$ \\
\hline 4 & $\begin{array}{l}\text { Pléniglaciaire } \\
\text { inférieur }\end{array}$ & E8 & & $\begin{array}{l}\text { Nappe de } \\
\text { solifluxion }\end{array}$ \\
\hline $\begin{array}{r}a \\
5 b \\
c \\
c\end{array}$ & Glaciaire ancien? & E9 & & Alluvions \\
\hline \begin{tabular}{|l|}
115 \\
$5 \mathrm{e}$
\end{tabular} & $\begin{array}{l}\text { Eémien } \\
\text { ou antérieur }\end{array}$ & E10 & & Altérites \\
\hline
\end{tabular}

Fig. 5: Stratigraphie synthétique du remplissage quaternaire des vallées sèches d'Ajoie

Synthetic stratigraphy of the Quaternary deposits filling the dry valleys of Ajoie

Stratigraphiesynthese von Quartären Füllungen in Trokkentälern der Ajoie

sommet comme un horizon d'altération développé in situ, on doit leur assigner un âge Glaciaire ancien (SI 5), soit antérieur à la formation de ces argiles décarbonatées, lesquelles pourraient être attribuées à la phase de pédogenèse de l'interstade d'Odderade (SI 5a) qui a conduit à la formation de sols lessivés dans le Fossé rhénan, le nord de la France et la Belgique (BoENigK \& Frechen 1999; Van Vliet-Lanoë \& Guillocheau 1995). En revanche, si l'on considère ce niveau argileux comme étant remanié, un âge Pléniglaciaire inférieur (SI 4) est envisageable. Notre préférence va pour l'instant à la première hypothèse, notamment en raison du fait que les stades de la péjoration climatique du Glaciaire ancien (SI 5b ou 5d) ont été encore relativement humides par rapport au climat plus aride du Pléniglaciaire ancien (VAN Vliet-LANö̈ \& GUILlocheaU 1995). L'épaisseur maximale observée des graviers E9 est de quatre mètres. Sur la base de données géophysiques et de forages, elle pourrait atteindre une dizaine de mètres au maximum.
Sont regroupées dans l'ensemble 10 des altérites dont la genèse n'est pas évidente à établir: terra fusca, argiles de décarbonatation ou encore horizons argiliques de pédocomplexes interglaciaires. D'âge éémien ou antérieures, elles se rencontrent au toit du bedrock karstifié ou dans les anfractuosités de l'épikarst.

\subsection{Paléohydrologie}

L'histoire sédimentaire et hydrologique qu'il est possible de reconstituer sur la base de l'enregistrement stratigraphique à disposition ne commence qu'au début de la dernière glaciation, plus précisément durant le Glaciaire ancien, il y a environ $115^{\prime} 000$ ans (Fig. 6). Des graviers fluviatiles (E9) se déposent alors dans le fond des vallées sèches d'Ajoie, tant en amont qu'en aval du Creugenat, en érodant et déblayant les dépôts meubles plus anciens jusqu'au substrat rocheux. L'environnement sédimentaire devait alors correspondre à un système de rivières en tresses à caractère torrentiel. C'est probablement vers la fin du Glaciaire ancien que le drainage de surface s'est enfoncé dans le karst, conférant à la Haute-Ajoie son statut de vallée sèche. Les archives sédimentaires ne livrent ensuite aucun indice d'écoulement superficiel pour les périodes du Pléniglaciaire inférieur et moyen durant lesquelles se déposent une nappe de solifluxion (E8) et des lœss ruisselés (E6/7). Il faut attendre le Pléniglaciaire supérieur pour voir des écoulements investir à nouveau les vallées sèches, en contexte périglaciaire sur sol gelé. De caractère torrentiel et vraisemblablement temporaire, ils ont déposé une nappe de graviers hétérométriques (E5). Cet épisode érosif a localement déblayé les dépôts plus anciens jusqu'au substrat. Durant le Tardiglaciaire, l'écoulement karstique souterrain semble avoir été prédominant. Les lœss altérés qui lui sont rattachés (E4) ont cependant été remaniés ultérieurement dans des chenaux. La datation de cet épisode fluviatile est malaisée, mais un âge situé entre 6'000 et 8'000 ans cal. BP peut être proposé, en croisant les données micromorphologiques, archéologiques et radiocarbone (DESLEX-SHEIKH et al. 2006).

Les dépôts humifères bruns à paillettes de charbons de bois rattachés aux ensembles sédimentaires E2 et E3 matérialisent un important impact anthropique sur les sédiments. La géométrie très irrégulière des chenaux observés en stratigraphie traduit un régime d'écoulement turbulent et irrégulier. Cette réactivation des écoulements de surface est peut-être à mettre en relation avec les défrichements anthropiques liés aux premières pratiques agricoles dans la région. En effet, la diminution de la couverture forestière a pu conduire, par augmentation du ruissellement de surface, à la réapparition temporaire de petits cours d'eau au fond des vallées sèches. Toutefois, la datation de ces épisodes fluviatiles a livré des âges qui parlent en faveur de 


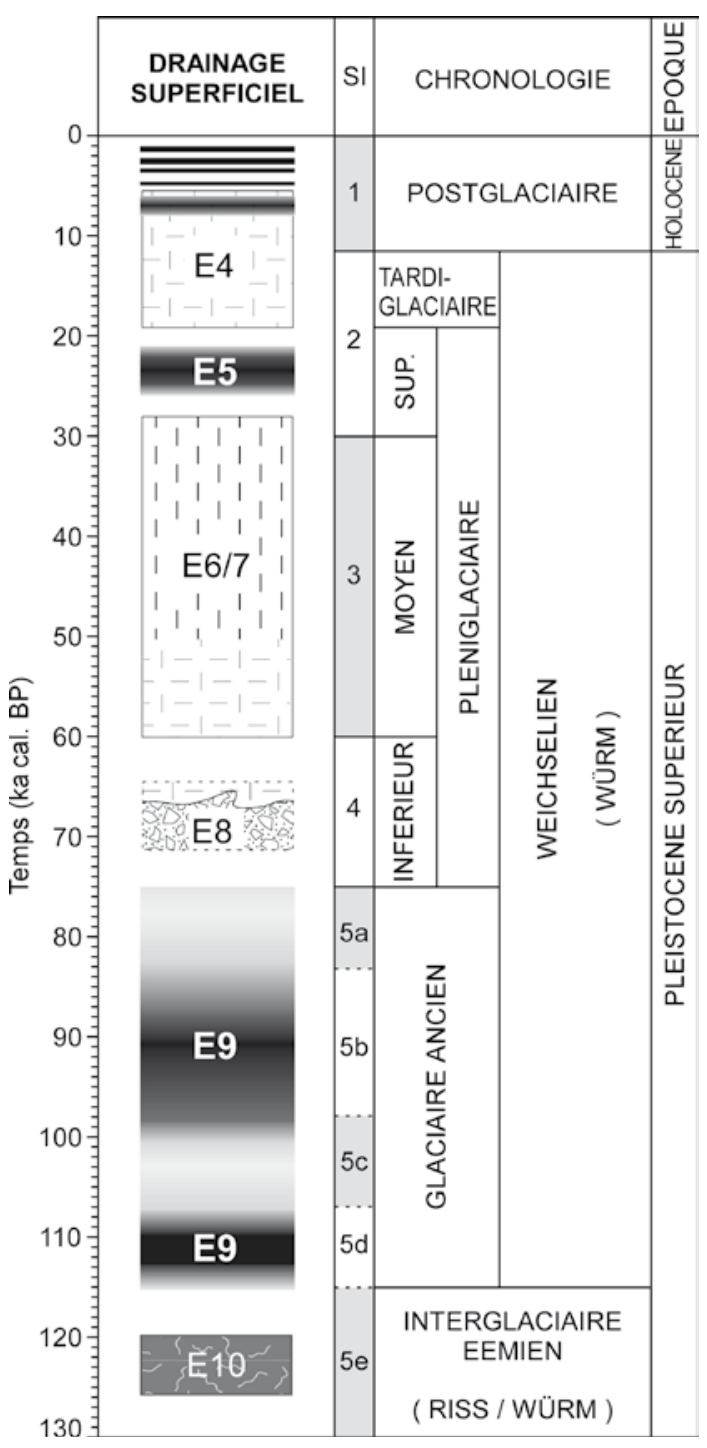

Fig. 6: Périodes de drainage superficiel (en noir) reconnues sur la base de la stratigraphie rencontrée dans le fond des vallées sèches d'Ajoie

Reconstructed periods (black) of superficial drainage in the dry valleys of Ajoie

Rekonstruierte Perioden (in schwarz) der oberflächligen Entwässerung in den Trockentälern der Ajoie

facteurs génétiques d'ordre climatique plutôt qu'anthropique. En effet, l'agencement des datations ${ }^{14} \mathrm{C}$ réalisées sur les charbons de ces alluvions met en évidence quatre épisodes de reprise de l'activité hydrologique dans les vallées sèches d'Ajoie depuis la fin du Néolithique (Fig. 7): Néolithique final (2'900-2'600 cal. BC); Bronze moyen (1'700-1'200 cal. BC); Hallstatt (-La Tène) (800-200 cal. BC); Haut Moyen Age (400-900 cal. AD). Ces épisodes d'activité fluviatile correspondent tous à des phases de haut niveau lacustre des lacs du Jura et du Plateau suisse (MAGNy 2004), ce qui suggère évidemment un contrôle climatique, en l'occurrence une augmentation des précipitations. D'un point de vue stratigraphique, le plus exacerbé de ces épisodes est celui du Haut Moyen Age. De manière tout à fait inattendue, il corrobore ainsi, en milieu karstique, la crise hydrique située entre 550 et 800 cal. AD mise en évidence dans le bassin molassique de Delémont (BRAillard 2008).

\section{Conclusions}

L'étude des sédiments meubles qui colmatent les vallées sèches d'Ajoie a révélé la présence d'un système de drainage fossile remontant pour l'essentiel au Glaciaire ancien weichsélien (SI 5), mais également actif au Pléniglaciaire supérieur (SI 2), sur pergélisol, ainsi que plus sporadiquement durant l'Holocène. Les épisodes fluviatiles holocènes se sont produits durant des périodes de hauts niveaux des lacs jurassiens (MAGNY 2004), ce qui suggère qu'une augmentation des précipitations en a constitué la cause. Toutefois, la pression anthropique sur l'environnement (dégradation de la végétation et des sols liée à l'agriculture) a certainement joué un rôle accessoire, ou peut-être déclencheur, dans un contexte climatique défavorable. Pour terminer, on relèvera le potentiel des vallées sèches comme indicateur paléohydrologique et paléoclimatique.

\section{Bibliographie}

Boenigk, W. \& M. Frechen (1999): Klimaschwankungen im Frühweichsel der Lößabfolgen des Mittelrheingebiets. - In: Eiszeitalter und Gegenwart 49: 124-131.

BraILlaRD, L. (2006): Morphogenèse des vallées sèches du Jura tabulaire d'Ajoie (Suisse): rôle de la fracturation et étude des remplissages quaternaires. - = GeoFocus 14, Fribourg: 1-224.

Braillard, L. (2008): La séquence palustre de Delémont, la Communance. - In: GuÉlAT, M., BrombaCHER, C., Olive, C., Wick, L. et al.: Develier-Courtételle, un habitat rural mérovingien. 4. Environnement et exploitation du terroir. $-=$ Cahier d'archéologie jurassienne 16, Porrentruy: 73-84.

Deslex-Sheikh, C., Saltel, S., Braillard, L. \& J. Detrey (2006): Le Campaniforme des vallées sèches d'Ajoie (JU): les sites de la combe En Vaillard et de la combe Varu à Chevenez. - In: Annuaire d'archéologie suisse 89: 51-86. 


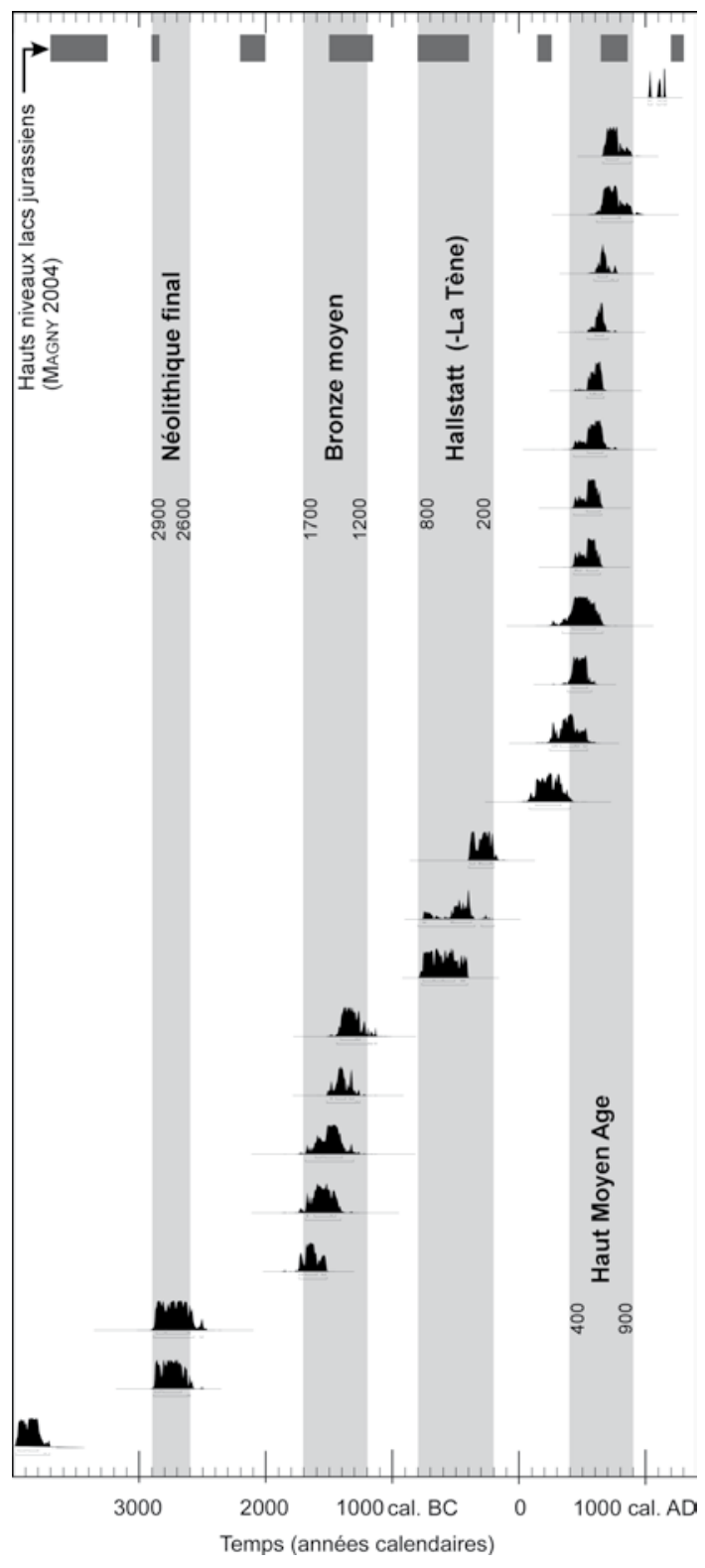

Fig. 7: Episodes fluviatiles dans les vallées sèches d'Ajoie depuis le Néolithique moyen

Fluviatile episodes in the dry valleys of Ajoie since the Middle Neolithic

Fluviatile Episoden in den Trockentälern der Ajoie seit dem mittleren Neolithikum
ERZINGER, E. (1943): Die Oberflächenformen der Ajoie (Berner Jura). - In: Mitteilungen der GeographischEthnologischen Gesellschaft in Basel IV: 1-138.

LièvRE, L. (1955): Etudes, recherches et travaux dans différents domaines des sciences naturelles en Ajoie, dans le Jura et les régions avoisinantes. - In: Recueil d'études et de travaux scientifiques, Porrentruy: 113170.

MagnY, M. (2004): Holocene climate variability as reflected by mid-European lake-level fluctuations and its probable impact on prehistoric human settlements. - In: Quaternary International 113, 1: 65-79.

Perronne, A. (1955): Vestiges des périodes glaciaires dans le Jura. - In: Recueil d'études et de travaux scientifiques, Porrentruy: 47-79.

Stuiver, M., Reimer, P.J., Bard, E., Beck, J.W., Burr, G.S., Hughen, K.A., Kromer, B., McCormac, G., VaN DER Plicht, J. \& M. SPURK (1998): IntCal98 radiocarbon age calibration, 24'000-0 cal BP. - In: Radiocarbon 40, 3: 1041-1084.

Van Vliet-Lanoë, B., Fagnart, J.-P., Langohr, R. \& A. Munaut (1992): Importance de la succession des phases écologiques anciennes et actuelles dans la différentiation des sols lessivés de la couverture lœssique d'Europe occidentale: argumentation stratigraphique et archéologique. - In: Science du sol 30, 2: 75-93.

Van Vliet-Lanoë, B. \& F. Guillocheau (1995): Evolution de l'enregistrement pédosédimentaire depuis 150 ka en France du NW et en Belgique: biorhexistasie et bilans sédimentaires. - In: Comptes Rendus de l’Académie des Sciences 320, IIa: 419-426.

\section{Résumé: Remplissages quaternaires et paléohydrolo-} gie des vallées sèches d'Ajoie (Jura tabulaire, Suisse) Le Jura tabulaire d'Ajoie est caractérisé par la présence de nombreuses vallées sèches. Afin de préciser leur morphogenèse, une étude multidisciplinaire a été conduite lors des travaux de prospection archéologique le long de l'autoroute A16 (BraILlard 2006). La section transversale des vallées sèches, en forme de $\mathrm{U}$, laissait supposer la présence de dépôts quaternaires en profondeur. Un remplissage de 2 à $10 \mathrm{~m}$ d'épaisseur a effectivement été mis au jour. L'étude stratigraphique détaillée de ce remplissage a permis une subdivision chronostratigraphique des dépôts en 10 ensembles (E1-E10): une couche très argileuse (E10) interprétée comme un horizon argilique éémien remanié, ou comme une altérite plus ancienne, est localement préservée sur le bedrock karstifié. Des graviers fluviatiles (E9, E5) constituent systématiquement la base du comblement. Ils témoignent d'un système de drainage fossile actif probablement durant le Glaciaire ancien weichsélien (SI 5), ainsi qu'au Pléniglaciaire supérieur (SI 2). Une nappe de solifluxion (E8), attribuée au Pléniglaciaire inférieur (SI 4), a été trouvée en pied de pente. Des lœess (E6/7) datés par OSL du Plénigla- 
ciaire moyen (SI 3) sont intercalés entre les graviers E9 et E5. Une partie de ces lœss (E4) a été remaniée et altérée durant le Tardiglaciaire et l'Holocène ancien. Finalement, quatre épisodes fluviatiles ont été repérés dans l'enregistrement sédimentaire holocène (E1 à E3). Leur corrélation avec des périodes de hauts niveaux des lacs jurassiens (MAGNY 2004) suggère qu'un contrôle climatique (augmentation des précipitations) est à l'origine de ces reprises temporaires de l'écoulement superficiel.

Mots-clés: Quaternaire, stratigraphie, paléohydrologie, vallées sèches, Jura tabulaire

\begin{abstract}
Quaternary fillings and palaeohydrology of the dry valleys of Ajoie (tabular Jura, Switzerland)

The tabular Jura of Ajoie is characterized by numerous dry valleys. In order to determine their morphogenesis, a multidisciplinary study was conducted during an archaeological excavation along the highway A16 (Braillard 2006). The U-shape transversal section of the dry valleys suggests the presence of Quaternary deposits under their flat bottom. Indeed, a 2 to 10 meter thick filling, containing fluviatile gravels at the base, was revealed. The detailed stratigraphic study of this filling allowed a chrono-stratigraphic subdivision of the deposits in 10 units (E1-E10): a reworked Eemien argillic horizon or an older alterite (E10) is locally preserved on the karstified bedrock. Fluviatile gravels (E9, E5) constitute the most important part of the filling. They testify to a fossil drainage system, active probably during the Weichselian Early Glacial (IS 5), and certainly during the Late Pleniglacial (IS 2). Solifluction deposits (E8), attributed to the Early Pleniglacial (IS 4), are locally present on footslopes. OSL-dated Middle Pleniglacial (IS 3) loess (E6/7) are intercalated between the two gravel bodies E9 and E5. Part of these loess (E4) were reworked and weathered during Late Glacial to Early Holocene. Finally, four fluviatile episodes were found in the Holocene sedimentary record (E1-E3). All of them correlate with known periods of Holocene high lake levels in the Jura (MAGNY 2004), and suggest that a climatic control (increase in precipitations) was responsible for the temporary reactivations of the superficial drainage.
\end{abstract}

Keywords: Quaternary, stratigraphy, palaeohydrology, dry valleys, tabular Jura

\section{Zusammenfassung: Quartäre Füllungen und Palaeo- hydrologie der Trockentäler der Ajoie (Tafeljura, Schweiz)}

Der Tafeljura ist durch zahlreiche Trockentäler charakterisiert. Um ihre Morphogenese präzisieren zu können, wurde eine multidisziplinäre Studie zusam- men mit einer archäologischen Bestandsaufnahme entlang der Autobahn A 16 durchgeführt (BRAILlard 2006). Der U-förmige Querschnitt der Trockentäler ließ das Vorkommen von quartären Sedimenten unter dem flachen Talboden vermuten. Tatsächlich wurden 2 bis $10 \mathrm{~m}$ mächtige, an der Basis aus fluviatilen Schottern bestehende Talfüllungen bestätigt. Die detaillierte Untersuchung der quartären Talfüllungen erlaubte eine chronostratigraphische Gliederung der Ablagerungen in zehn Einheiten (E1-E10): Ein umgelagerter Eem-zeitlicher Tonhorizont oder ein älterer Verwitterungshorizont (E10) ist lokal über dem verkarsteten Grundgestein erhalten. Fluviatile Kiese (E9, E5) bilden systematisch die Basis der Talfüllungen. Dies zeugt von einem fossilen Abflusssystem, das während des Weichsel-Frühglazials (Frühwürm, IS 5) sowie im Verlauf des Oberen Pleniglazials (IS 2) aktiv war. Solifluktions-Ablagerungen (E8) des Frühen Pleniglazials (IS 4) finden sich lokal am Hangfuß. Lösse (E6/7), die anhand von OSL-Messungen ins Mittlere Pleniglazial (IS 3) datiert wurden, sind zwischen den Kieskörpern E9 und E5 eingeschaltet. Teile dieser Lössablagerungen (E4) verwitterten während des Spätglazials und Holozäns und wurden umgelagert. Abschliessend sind vier fluviatile Episoden für das Holozän dokumentiert (E1-E3), die mit bekannten holozänen Hochständen der Seespiegel im Jura korrelieren (MAGNY 2004). Dies könnte auf klimatische Ursachen zurückzuführen sein (Zunahme der Niederschläge).

Schlüsselwörter: Quartär, Stratigraphie, Palaeohydrologie, Trockentäler, Tafeljura

Dr. Luc Braillard, Département des Géosciences, Section de géologie, Université de Fribourg, Chemin du Musée 6, CH-1700 Fribourg, Suisse.

e-mail: luc.braillard@unifr.ch

\section{Manuskripteingang/received/manuscrit entré le 5.11.2008}

Annahme zum Druck/accepted for publication/accepté pour l'impression: 4.9.2009 